\title{
Experimental combustion chamber simulation at transient regimes
}

\author{
Bogdan Gherman ${ }^{1, *}$, Ion Mălăel ${ }^{1}$, Florin Florean $^{1}$, and Ionuț Porumbel ${ }^{1}$ \\ ${ }^{1}$ Romanian Research and Development Institute for Gas Turbines - COMOTI, bl. Iuliu Maniu, no. 220D, district 6, Bucharest, Romania
}

\begin{abstract}
The transient regimes in a combustion chamber has to be as short as possible because flame front position and thickness can destroy the combustion chamber in couple seconds. The simulation of such a regime has to be performed unsteady. An experimental combustion chamber it is simulated at two unsteady regimes to see the flame front structure and comparison it is made with the experimental data to validate the results. For this analysis Ansys CFX was used and the turbulent model was DES while the combustion model was Eddy Dissipation. The two cases show different flame front structures while the boundary conditions for the two regimes are very similar.
\end{abstract}

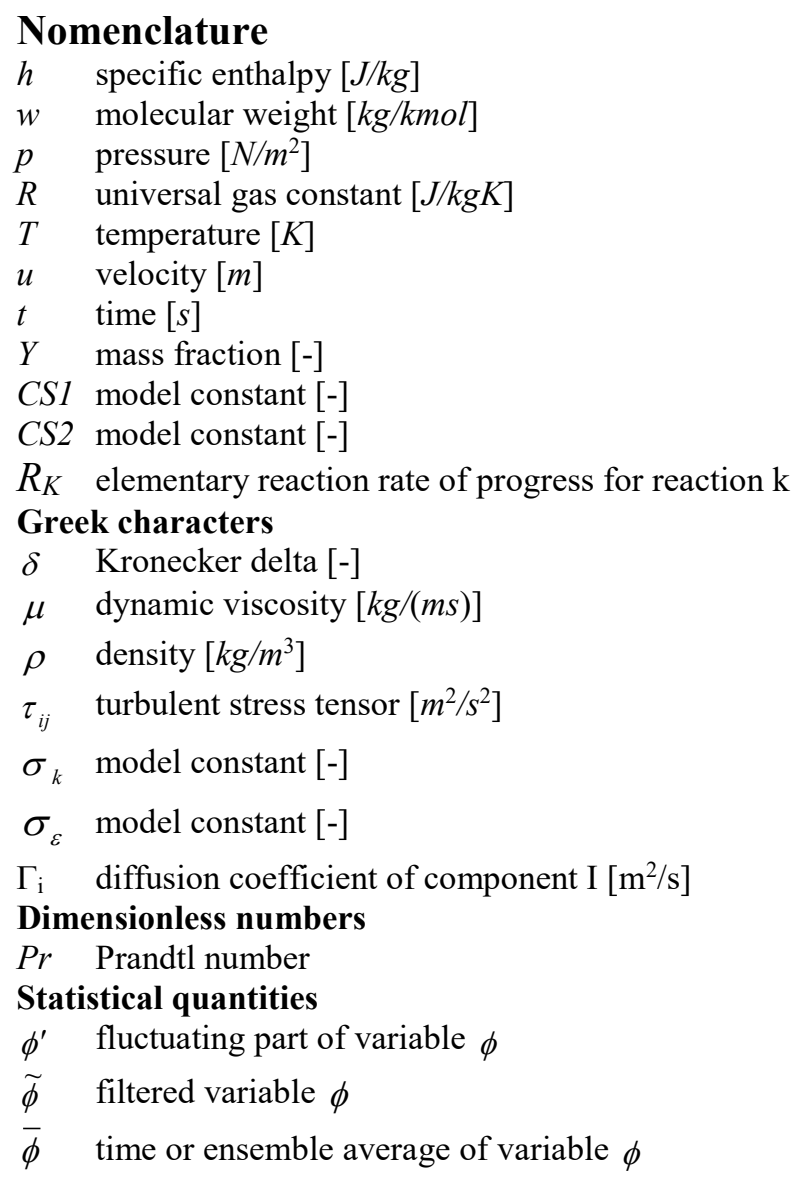

\section{Introduction}

The main problem of a combustion chamber is the flame stability at different operational regimes. The flame can be stabilized inside a combustor by two methods: bluff body [1] or recirculation zones [2]. The other important aspect inside a combustion chamber is the mixing of fuel and oxidizer prior to combustion [3], which could improve the combustion efficiency and can decrease pollutant emissions [4], [5]. Also, an unsteady flame front can affect the turbine stator by delivering a nonuniform flow field at the turbine entrance [5] and this can lead to the formation of hot spots that can damage the turbine. Not only that but an oscilating flame front can attach to the combustion walls and in this situation the combustion chamber can be destroyed in very short time [6], [7]. That is why when a combustion chamber is developed it is very important to test it to all stationary and transient regimes that may be attained and asses the impact on the combustor geometry and performances.

In this study it is performed a numerical analysis of two transient regimes of an experimental combustion chamber.

\section{Problem Description}

The experimental campaign that concern the combustion chamber testing was performed to test a sensor for velocity and temperature measurements. The combustion chamber was tested at different regimes to asses the capability of the sensors to accurately measure velocity and temperature.

In this paper is presented the numerical simulation of the experimental combustion chamber from an industrial micro-gas turbine, at two transient regimes chosen based on the fuel mass flow, thus the first regime is with approximately $30 \%$ higher than the reference mass flow and the second case have the fuel mass flow with $30 \%$ lower than the nominal mass flow. Due to the extensive experiments performed on this combustion chamber it is possible to simulate only a small part of the experimental installation. In the figure below can be seen the two geometries.

\footnotetext{
* Corresponding author: bogdan.gherman@comoti.ro
} 


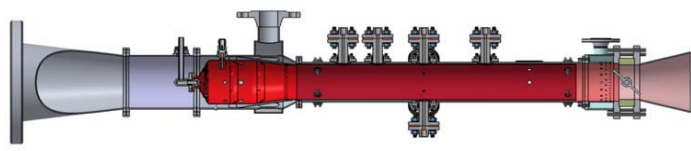

$\mathrm{a}$

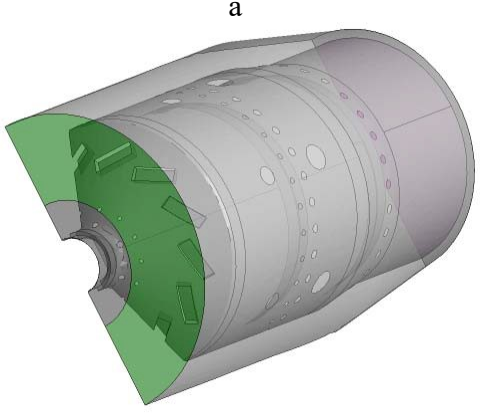

b

Fig. 1. The combustion chamber ensemble: experimental rig (a) and the simulated geometry (b).

To prepare the combustion chamber for simulation some features of it had to be removed. Like thickness of the walls, screw and bolts had to be removed. This operation does not influence the flow inside the combustion chamber. Also due to a periodicity of the combustion chamber it was possible to simulate only half of it as it can be seen in Fig. 2.

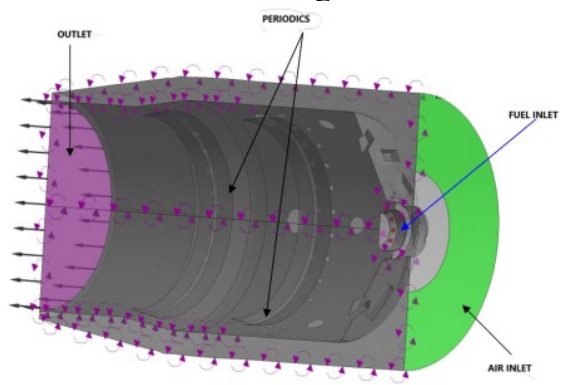

Fig 2. Computational domain with boundary conditions.

These operations were necessary to reduce the computational time and the number of cells. Due to the possibility to have experimental data close to the combustion chamber, it was also possible to cut the domain very short. The injector geometry was adapted to this analysis because the flow inside the injector does not concern the current study. As a consequence, only the outer shape of it is discretize and the boundary conditions are imposed at the exit of the injector as it can be seen in Fig. 3.

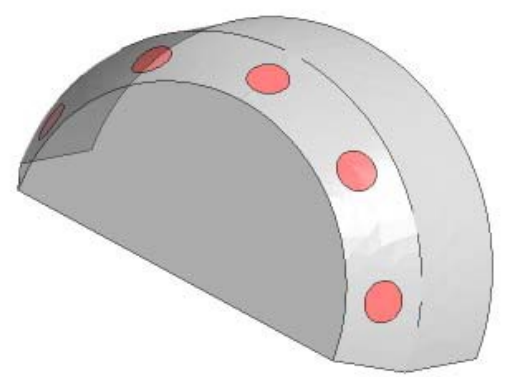

Fig. 3. Injector geometry.

\subsection{Domain Discretization}

The resolution of the grid had to consider the sensitive areas of the domain. Close the injector inlets the cell size is 0.2 or less and around the first part of the domain, where the flame front it is situated the grid size is around $0.4 \mathrm{~mm}$ or less. The growth rate of the cell size is around $1.2 \sim 1.3$.

In total have been used approximately 4.3 million cells for this half of the combustion chamber.

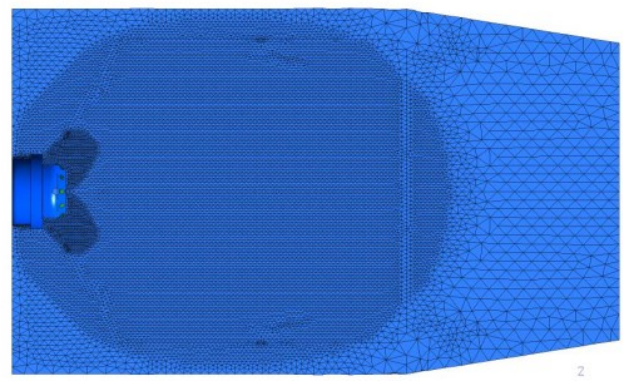

Fig. 4. Cell distribution in the domain.

\subsection{Boundary conditions and analysis type}

There are two cases that are studied here, each case with the following boundary conditions:

Case 1:

Air inlet mass flow rate: $3.0826 \mathrm{~kg} / \mathrm{s}$;

Fuel inlet mass flow rate: $2.04 \mathrm{e}-2 \mathrm{~kg} / \mathrm{s}$;

Outlet pressure: 10.2 bar.

Case 2:

Air inlet mass flow rate: $3.937034 \mathrm{~kg} / \mathrm{s}$;

Fuel inlet mass flow rate: $0.011414 \mathrm{~kg} / \mathrm{s}$;

Outlet pressure: 10 bar.

Zero velocity was imposed at the walls and they are adiabatic.

Due to unsteady behaviour of the flame front for this study was considered a compressible flow, unsteady, the equations are written in Reynolds Averaged form below [8]:

1. The Continuity Equation:

$$
\frac{\partial \bar{\rho}}{\partial t}+\frac{\partial \bar{\rho} \tilde{u}_{j}}{\partial x_{j}}=0
$$

2. The Momentum Equations:

$$
\frac{\partial \bar{\rho} \tilde{u}_{i}}{\partial t}+\frac{\partial \bar{\rho}\left(\tilde{u}_{i} \tilde{u}_{j}\right)}{\partial x_{j}}=-\frac{\partial \bar{p}}{\partial x_{i}}+\frac{\partial}{\partial x_{j}}\left(\overline{\tau_{i j}}-\overline{\rho u_{i}^{\prime} u_{j}^{\prime}}\right)
$$

where:

$$
\overline{\tau_{i j}}=\mu\left[\frac{\partial \tilde{u}_{i}}{\partial x_{j}}+\frac{\partial \tilde{u}_{j}}{\partial x_{i}}-\frac{2}{3} \delta_{i j}\left(\frac{\partial \tilde{u}_{k}}{\partial x_{k}}\right)\right]
$$

represents the stress tensor.

3. The Total Energy Equation:

$$
\begin{aligned}
& \frac{\partial}{\partial t}(\bar{\rho} \tilde{h})+\frac{\partial\left(\bar{\rho} \tilde{u}_{j} \tilde{h}\right)}{\partial x_{j}}=\frac{\partial \bar{p}}{\partial t}+\frac{\partial}{\partial x_{j}}\left(\frac{\bar{\mu}}{\operatorname{Pr}} \frac{\overline{\partial h}}{\partial x_{j}}\right)+ \\
& +\overline{\tau_{i j}} \frac{\partial \tilde{u}_{j}}{\partial x_{j}}+\frac{\partial}{\partial x_{j}}\left(-\overline{\rho h^{\prime} u_{j}^{\prime}}\right)
\end{aligned}
$$

where $\mathrm{h}$ is the enthalpy. 
4. Scalar transport Equation:

$$
\begin{aligned}
& \frac{\partial\left(\bar{\rho} \widetilde{Y}_{I}\right)}{\partial t}+\frac{\partial\left(\bar{\rho} \widetilde{u}_{j} \widetilde{Y}_{I}\right)}{\partial x_{j}}=\frac{\partial}{\partial x_{j}}\left(\Gamma_{I_{e f f}} \frac{\partial Y_{I}}{\partial x_{j}}\right)+S_{I} \\
& \widetilde{Y}_{I}=\frac{\widetilde{\rho}_{i}}{\bar{\rho}}, S_{I}=W_{I} \sum_{k=1}^{K}\left(v_{k I}^{\prime \prime}-v_{k I}^{\prime}\right) R_{K}
\end{aligned}
$$

5. Ideal Gas Equation of State:

$$
\widetilde{\rho}=\frac{w\left(\widetilde{p}+p_{\text {ref }}\right)}{R_{0} \widetilde{T}}
$$

where, $w$ is the molecular weight.

As a turbulence model it was considered the DES model (Detached Eddy Simulation). The idea behind this model is that when the turbulence length $\mathrm{L}_{t}$ is bigger than the local grid size then the analysis begins using LES instead of SST - RANS. In this case the length scale used for dissipation rate in the kinetic turbulent energy equation it is replace by $\Delta$, local size of the grid.

$$
\begin{aligned}
& \varepsilon=\beta^{*} \cdot k \cdot \omega=k^{\frac{2}{3}} / L_{t} \rightarrow k^{\frac{2}{3}}\left(C_{D E S} \cdot \Delta\right) \\
& C_{D E S} \cdot \Delta\left\langle L_{t}\right. \\
& \Delta=\max \left(\Delta_{i}\right) ; L_{t}=(\sqrt{k}) / \beta^{*} \cdot \omega
\end{aligned}
$$

The practical reason for the usage of the grid size is to be able to use the RANS close to the walls while in the other parts of the domain to use LES, which captures better the structures of the flow.

Also, as a combustion model it was used Eddy Dissipation combustion model. The majority of fuels burn very quickly and for this reason the chemical reaction speed is controlled by the reactants turbulent mixing. In the cases where the reactants are not premixed, turbulence helps driving/mixing slow the fuel and oxidizer toward reaction zones where burns quickly.

This model is a very robust combustion model that is good when it is used one global chemical reaction. If it is used in cases with more than one chemical reaction the results yield with this model will not be accurate because these reactions will be based on the Arrhenius relation that differs from one reaction to another.

\section{Results and Discussions}

The mixing process inside the combustion chamber for the first case shows that the stoichiometric ratio it is attained at a safe distance from the injector, thus the flame front develops away from the walls and injector (see Fig.5).

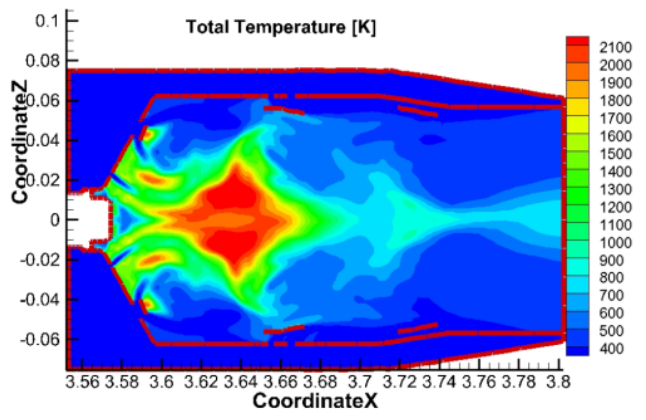

Fig. 5. Temperature distribution inside the combustion chamber for case 1 .
However, there are small pockets of high temperature attached to the combustion geometry as it can be seen in the figure above.

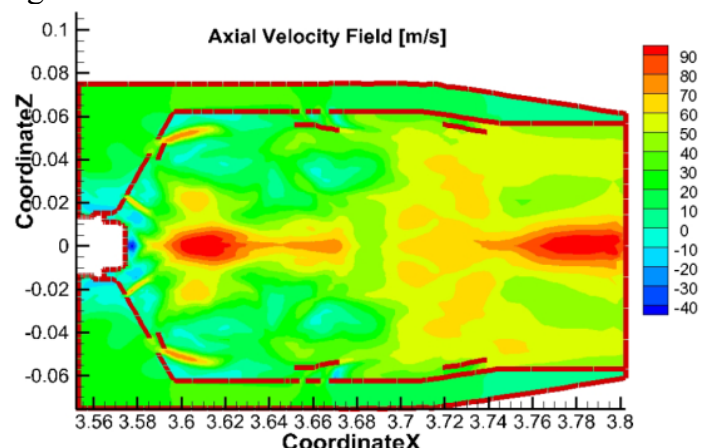

Fig. 6. Velocity distribution inside the combustion chamber for case 1 .

The axial velocity distribution plot, Fig. 6, shows a high velocity region at the outlet of the combustion chamber and another one close to the injector, prior to combustion.

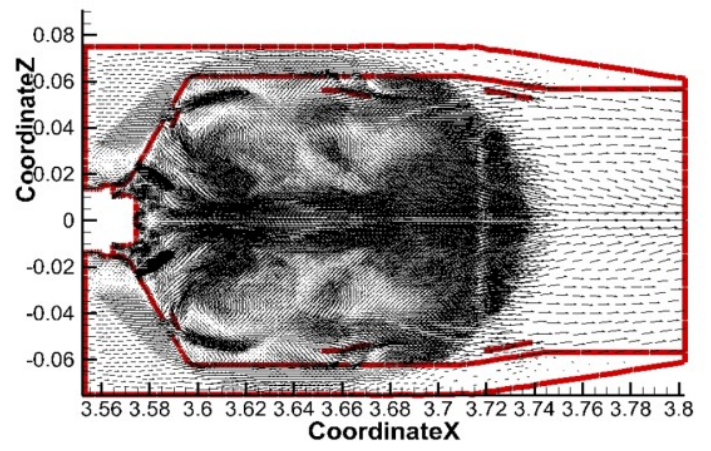

Fig. 7. Vector distribution inside the combustion chamber for case 1 .

This high velocity region helps mixing between air and fuel. The developed recirculation zones seen in the first part of the combustion chamber helps anchor the flame there as it can be seen in Fig. 9.

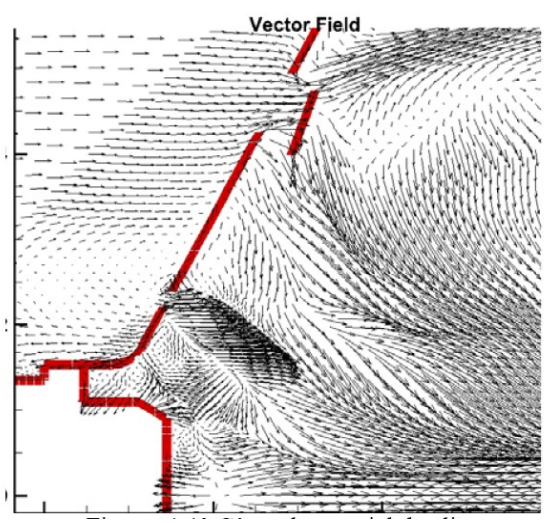

Fig. 8. Vector distribution close to the injector.

The high turbulence region that develops close to the injector moves the fuel away from the injector and does not allow the stoichiometric ratio to be attained so close to the injector wall, see Fig. 8.

In the second case studied the flame front it is still at a safe distance from the walls of the combustion chamber and injector. However, as it can be seen in Fig. 9 there are no more pockets with high temperature. 


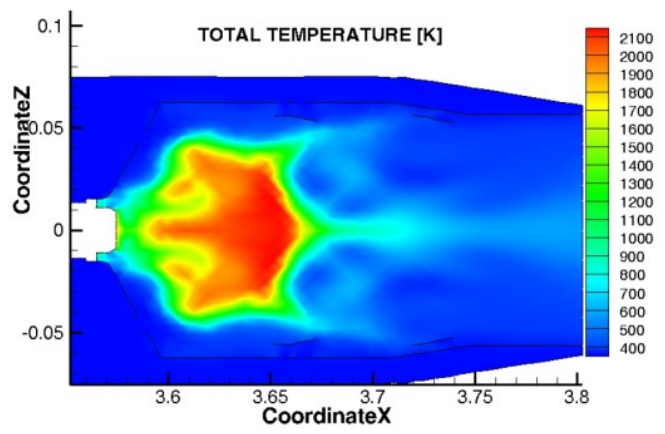

Fig. 9. Temperature distribution inside the combustion chamber for case 2 .

The axial velocity plot shows a more uniform flow field at the exit of the combustion chamber, see Fig. 10. Also, there is a high velocity region where the flame front is located.
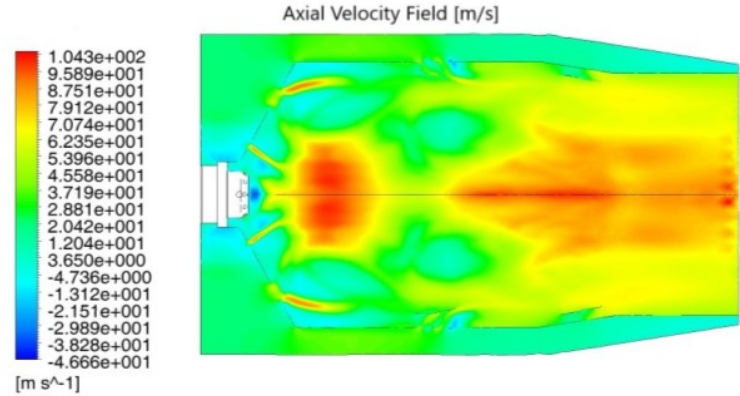

Fig. 10. Velocity distribution inside the combustion chamber for case 2 .

The vector field presented in Fig. 11, 12 shows high turbulence regions similar to the other case.

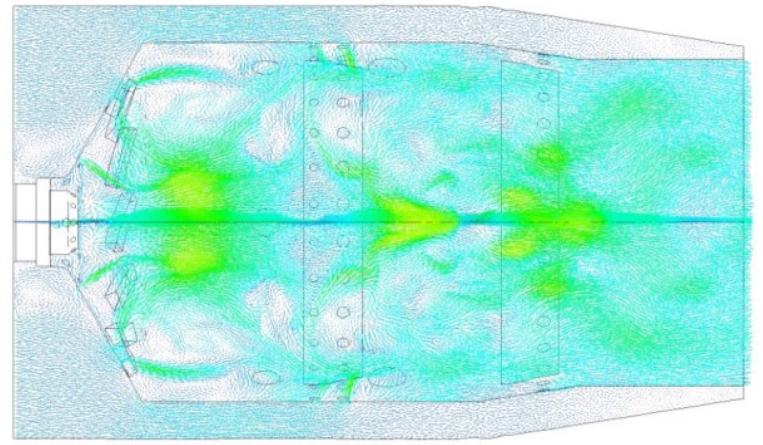

Fig. 11. Vector distribution inside the combustion chamber for case 2 .

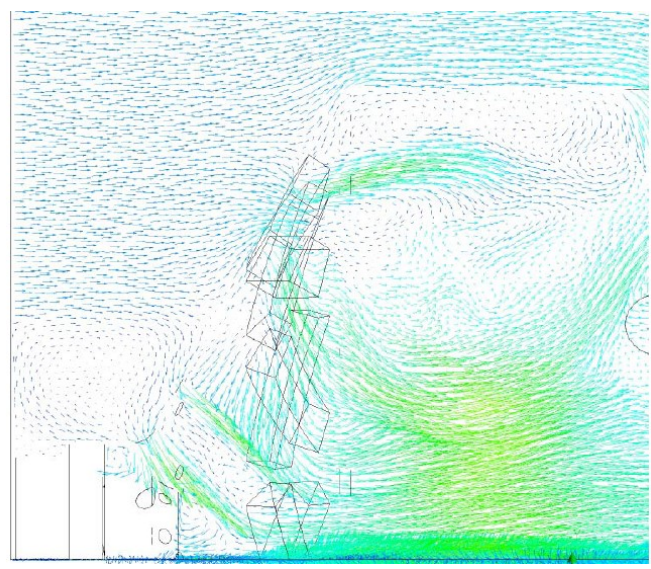

Fig. 12. Vector distribution close to the injector, case 2.
Also, to quantify the differences between the two cases five measurement line where extracted from the results file. The first line is close to the injector inlet, second line immediately after conical part of the combustion chamber, the third line it is situated at the end of combustion zone, the forth line it is situated at the exit from the combustion chamber, as it can be seen in Fig. 13. The last line is taken in the middle of the combustion chamber along streamwise direction.

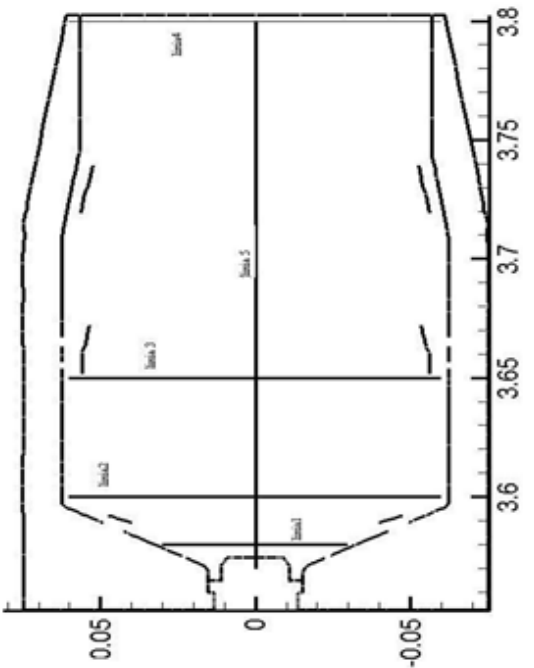

Fig. 13. Measurement lines positions inside the combustion chamber.

The velocity profile at line one shows that in the middle of the injector there is a recirculation zone that forms and can be seen in Fig. 13, Fig. 6 and Fig. 10. Also, it can be seen also the air from the cooling holes in the combustion tube that are around the injector, see Fig. 2 . The velocity profiles differ in intensity due to higher mass flow rate in the first case.
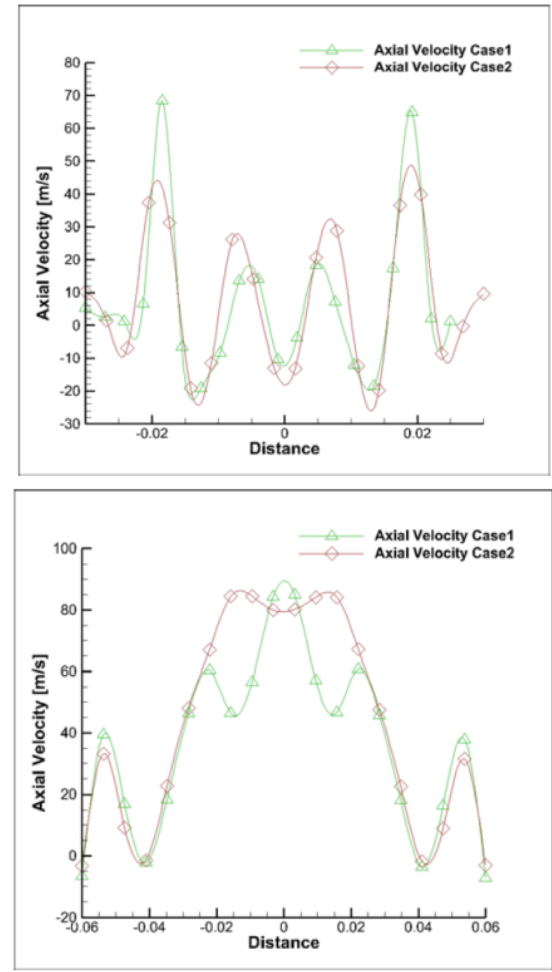

Fig. 13. Axial velocity at first line and second line. 
At the second line it can be observed that in the second case fuel jet is mixed, while the jet from the cooling holes around the injector it can be seen still.
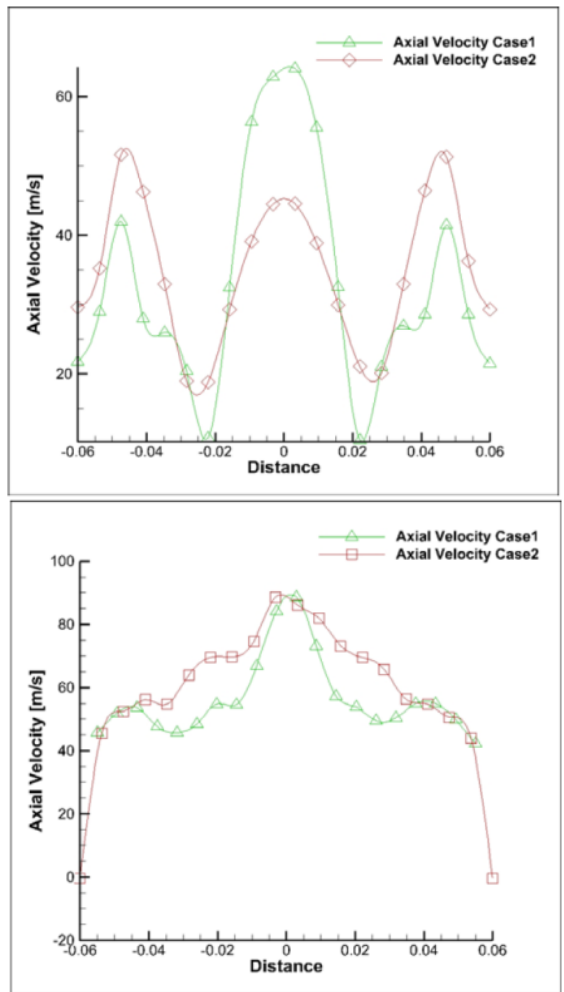

Fig. 13. Axial velocity at third and fourth line.

In fig. 13 at the end of the combustion zone it can be seen close to the walls the cooling air that forces the flame front to stay in the middle of the combustion chamber. Also, there are differences between the two cases, the velocity is higher in the first case where the mass flow rates are higher. At the exit of the combustion chamber in the second case the velocity profile shows a smooth increase in velocity.

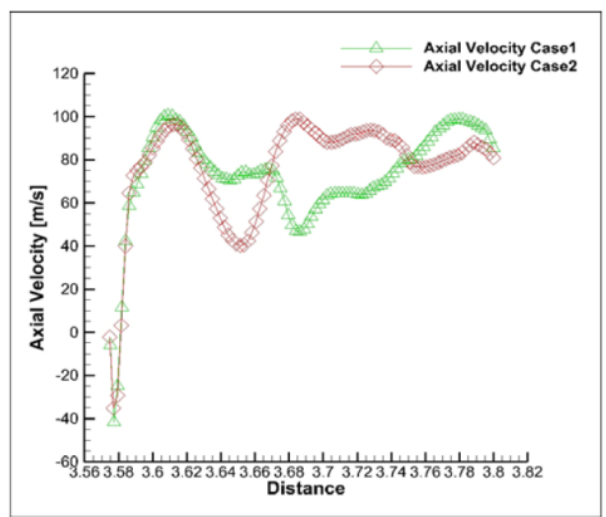

Fig. 14. Axial velocity at fifth line.

Along the streamwise direction it can be seen also the recirculation zones close to the injector, see Fig. 14, 6 and 10. It is interesting to notice that a decrease of velocity it is seen exactly where the flame front is situated in case 2 and after that the velocity it is rather constant. In the first case the velocity profile is more divers with multiple areas of acceleration and deceleration.

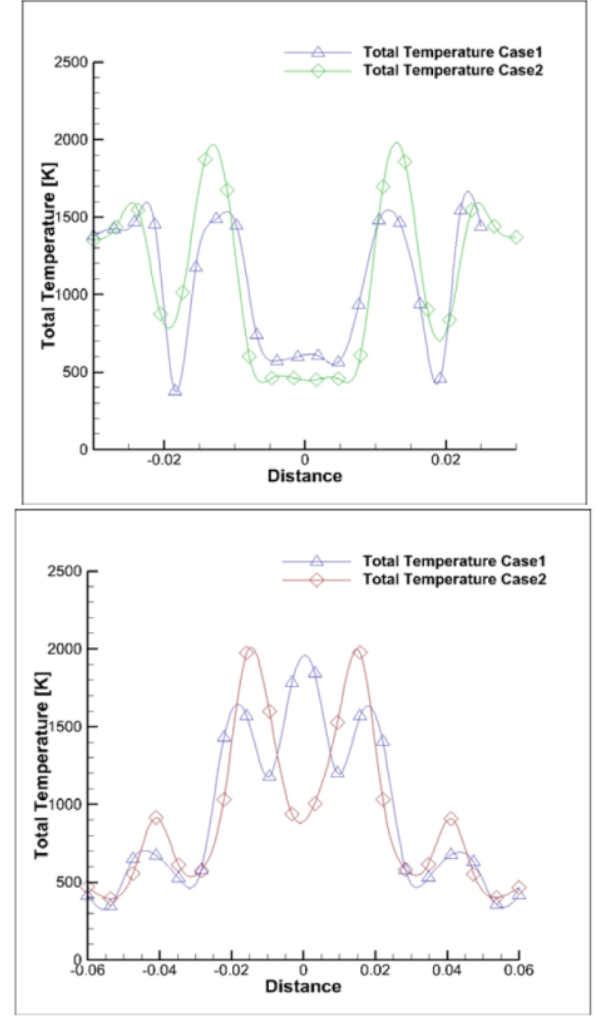

Fig. 15. Temperature at first and second line.

The temperature profile at the first line shows that there are pockets of high temperature close to the injector. In the second line it is noticeable that the flame it is developing but close to the walls the cooling air manages to keep the flame in the middle of the combustion chamber away from the walls, see Fig. 15, 5 and 9.
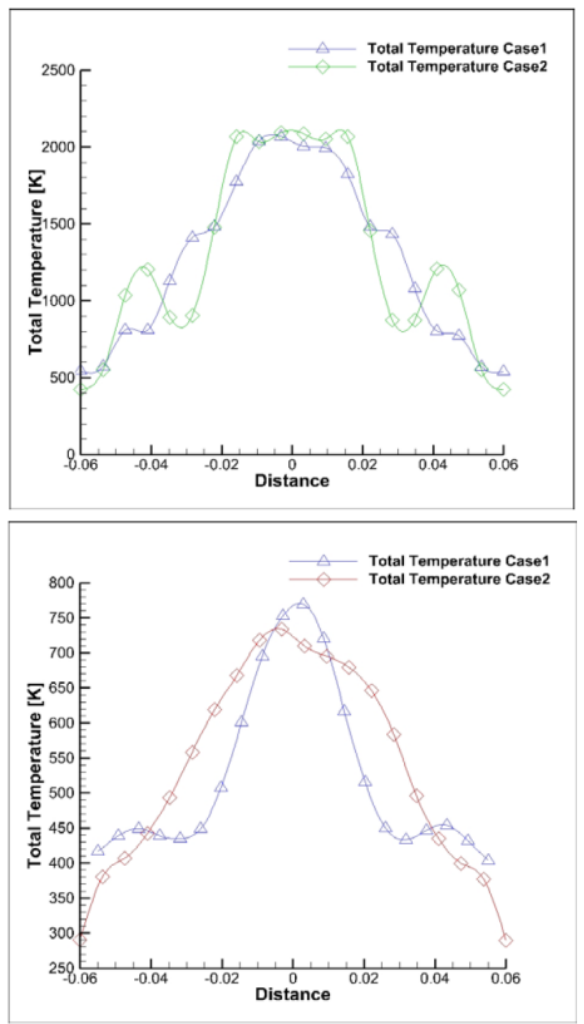

Fig. 16. Temperature at third and fourth line. 
The temperature profile for the third line shows again that close to the combustion chamber walls the temperature is around $500 \mathrm{~K}$ and at the forth line the temperature close to the walls is approximately $400 \mathrm{~K}$ for case 1 and around $273 \mathrm{~K}$ for the second case. In the second case the cooling air is more efficient.

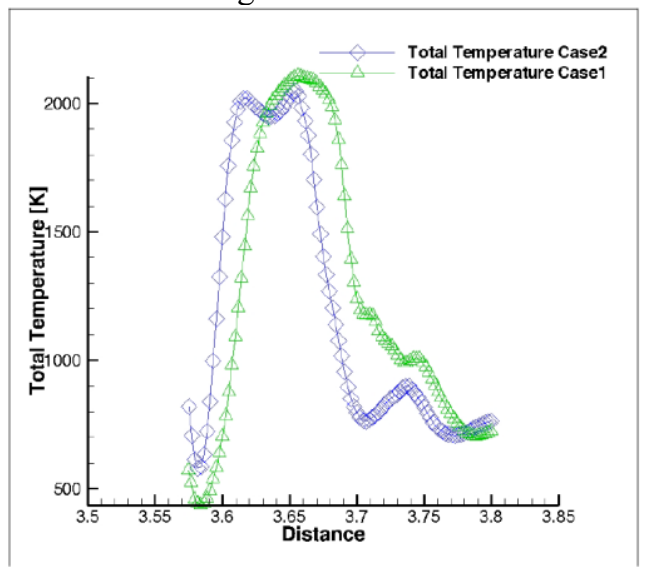

Fig. 17. Temperature at fifth line.

The temperature profile along the streamwise direction show the flame position that differs between the two cases, Fig. 17. It is noticeable that as soon as the fuel mass flow rate is decreased, the flame gets closer to the injector, thus the stoichiometric ratio it is attained much faster.

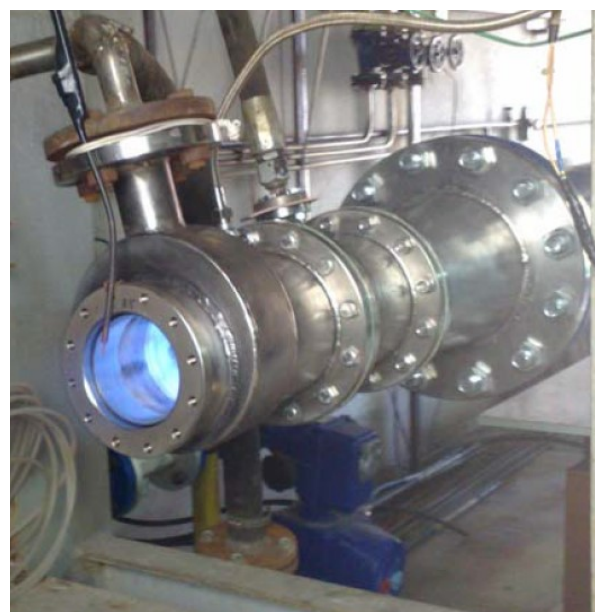

Fig. 18. Combustion test rig during tests.

The measured temperature by the four thermocouples at the exit of the combustion chamber are the following:

Case 1: 343402460324 [K]

Case 2: 320343393304 [K]

It has to be said that the four thermocouples measure the temperature near the walls. That is why the comparison between the values mentioned above and the graph in Fig. 16, fourth line, will have to be compared close to the walls. According to both sets of data, experimental and numerical it can be seen that it is a good correlation between them which shows that what it was noticed numerically, probably happened inside the combustion chamber during these transient regimes.

\section{Conclusions}

In this study it was performed an unsteady analysis of two transient regimes inside a combustion chamber. The analysis shows the problems that may appear in such situations. As it was noticed in the first regime studied there are visible hot spots close and attached to the walls of the combustion chamber that can damage irreversibly the structure. Also, it is noticeable the presence of high temperature regions in the graphs displayed in that paper close to the injector. Also according the experimental results there is a good match with the numerical simulations that shows that it is possible that what it was noticed numerically, probably happened inside the combustion chamber during these transient regimes.

\section{References}

1. Florean F., Porumbel I., Gherman B., Dumitrascu

$\mathrm{G}$-Experimental Measurements And Numerical Simulations In Bluff Body Stabilized Flames, NCAS, first international conference New challenges in aerospace science, Bucharest, 2015.

2. Gherman, B.G., Szasz, R.Z., Fuchs, L., LES of Swirling flows in Gas Turbine combustion chambers, ASME Turbo Expo 2004, Vienna, Austria, June, 2004.

3. A. C. Petcu, B. Gherman, F. G. Florean, C. Sandu, I. Porumbel - NUMERICAL SIMULATIONS OF ROUND TURBULENT JET FLAMES, NonEquilibrium Processes in Plasma Combustion and Atmosphere, Publisher: Torus Press, Editors: A.M. Stavrik, S.M. Frolov, pp.395 - 401, 201, ISBN 978-594588-121-1, Russia, 2012

4. B. Gherman, V. Stanciu - Influence of the flame front on the flow inside a combustion chamber - ISSN 1454-2358, U.P.B. Sci. Bull., Series D, Vol. 74, Iss. 3, 2012

5. M. Enache, A.C. Mangra, R. Carlanescu, F.G. Florean, The design of an annular combustion chamber, Aerospace Europe CEAS 2017 Conference, Bucharest, 2017

6. B. Gherman - Numerical Modeling of Gas Turbine Related Combustion - ISRN LUTMDN/TMHP04/7021-SE, 2005

7. A.C. Mangra, I. Porumbel, F.G. Florean, Experimental measurements of Camelina sativa oil combustion, Energy for Sustainable Development Journal, vol. 44, pg. 109-116, 2018

8. CFX Limited, Waterloo, Ontario, Canada, CFXTASCflow Theory Documentation, Section 4.1.2, Version 2.12, 2002 